\title{
Rare Oral Mucosal Manifestations of Hydroxyurea Therapy in Chronic Myeloid Leukemia
}

\author{
Deena Mudawi*, Abdulqadir J Nashwan, Amna Gameel, Shehab \\ Fareed, Mohammad Abdulla and Mohamed A Yassin* \\ Hematology Section, National Center for Cancer Care and Research, Qatar
}

*Corresponding authors: Deena Mudawi and Mohamed A Yassin, Consultant

\section{Review Article \\ Volume 1 Issue 2}

Received Date: September 04, 2017

Published Date: October 27, 2017

DOI: $10.23880 / \mathrm{hij}-16000107$

Hematologists and Assistant Professors of Clinical Medicine, WCMCQ, Qatar, E-mail: Yassin@hamad.qa

\section{Abstract}

Chronic myeloid leukemia (CML), is myeloproliferative neoplasm, that accounts for $15-20 \%$ of all leukemia's in adults, resulting from reciprocal translocation between the long arms of chromosomes 9 and 22(Philadelphia chromosome), producing BCR-ABL oncogene fusion, which is currently targeted by tyrosine kinase inhibiters (TKIs), but still Hydroxyurea (HU) usedin chronic myeloid leukemia, its role as cytoreduction and as bridging therapy before starting tyrosine kinase inhibitors (TKI).

There are several recognized side effects of hydroxyurea, such as cutaneous abnormalities and here we report rare mucous manifestations of hydroxyurea.

Keywords: Rare oral mucosal; Chronic myeloid leukemia; Hydroxyurea

Abbreviations: CML: Chronic Myeloid Leukemia; TKI: Tyrosine Kinase Inhibiters; HU: Hy-droxyurea

\section{Case Report}

We report 29-year-old female previously healthy, not on regular medications presented to emergency room with seven days history of fatigue and abdominal fullness. Her clinical examination revealed enlarged spleen $7 \mathrm{~cm}$ below costal margin. Investigations showed White blood counts 213,000 U/L (4-10,000 U/L) hemoglobin $10.1 \mathrm{~g} / \mathrm{dl}(12-15 \mathrm{~g} / \mathrm{dl})$,platelets count $266,000 \mathrm{U} / \mathrm{L} \quad(150-450,000 \mathrm{U} / \mathrm{L})$, peripheral blood shows moderate normochromic anemia with neutrophilic hyperleukocytosis with shift to left, eosinophilia, basophilia, monocytosis and $2 \%$ circulating blasts.

The patient underwent bone marrow aspiration and biopsy. Bone marrow aspirate shows marked myeloid hyperplasia and $2 \%$ blasts by differential count. Bone marrow biopsy is extremely hypercel-lular with $100 \%$ cellularity and showing marked myeloid hyperplasia and decreased erythropoiesis. Megakaryocytes are of uneven distribution with some small hypolobated forms noted CD -34 IHC stain shows no increase in positivity. FISH analysis revealed an abnormal hybridization signal pattern with dual fusion indicating BCR/ABL1 rearrangement, $t(9 ; 22)$ in $194 / 200$ cells analyzed. These findings are consistent with Chronic Myelogenous Leukemia (CML) in chronic phase. Patient started on hydroxyurea for 2 weeks as cytoreduction, developed hyper pigmented lesions on her tongue, see Figure 1. Hydroxyurea stopped for 2 weeks after which the pigmentation resolved, see Figure 2.

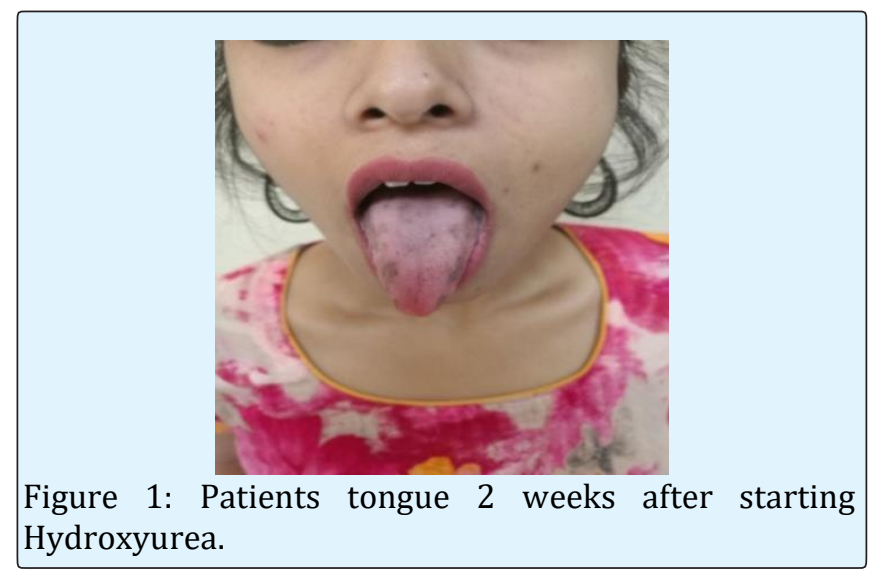




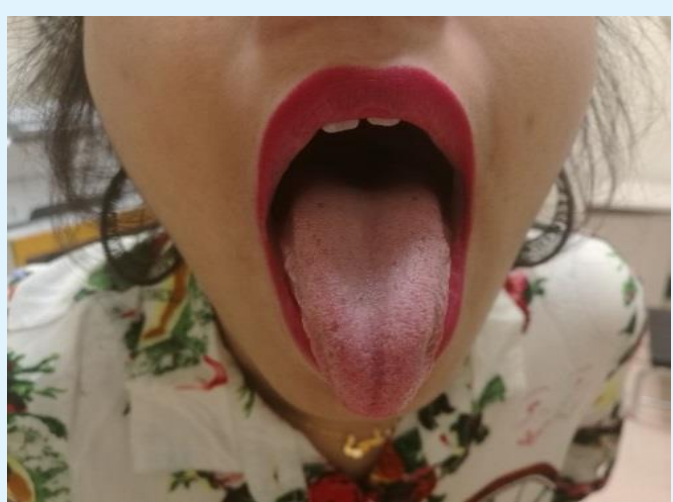

Figure 2: Patients tongue 2 weeks after stopping Hyroxyurea.

\section{Discussion}

Hydroxyurea (HU) has multiple usages in hematology with different mechanisms of actions. In sickle cell disease it acts by inducing the production of $\mathrm{HbF}$ [1], while in acute and chronic leukemia's it is used as cytoreduction. In chronic myeloid leukemia, hydroxyurea as well as its role as cytoreduction, it is used as bridging therapy before starting tyrosine kinase inhibitors (TKI) [2]. There are several recognized side effects of hydroxyurea, such as Cutaneous abnormalities [3] include xerosis, ichthyosiform lesions, dark brown pigmentation of skin folds and nails, malleolar ulcers as well as malignant tumors and mucosal pigmentations(reference and Beaus lines) [3].

On the other hand, oral lesions are much rarer and often present as painful ulcers associated with skin lesions [4]. Glossitis and stomatitis with intense erythema, fissuring and flattening of mucosal papillae may also be observed [5]. These lesions can appear right after the beginning of HU therapy or can be a late effect. Our patient developed oral mucosal hyper pigmented lesions one week after starting HU. The presence of particularly symptomatic oral lesions may indicate the need for dose reduction or treatment withdrawal, since these approaches lead to symptom improvement or resolution of the lesions [5]. In our case, oral hyper pigmented lesion in setting of CML and hydroxyurea never reported or extra ordinary rare.

\section{Conclusion}

Patients undergoing HU, especially those with myeloproliferative neoplasms (chronic myeloid leukemia) should have oral examination prior and during the therapy, since early recognition of alterations allows the reduction of comorbidities related to disease or therapy. In addition, presence of oral lesions may be an indication for dose reduction, discontinuation or replacement of therapy.

\section{References}

1. Rohit KA, Rakesh KP, Varsha S, Lalit N, Bhadra T (2014) Hydroxyurea in Sickle Cell Disease: Drug Review. Indian J Hematol Blood Transfus 30(2): 9196.

2. Hehlmann R, Heimpel H, Hasford J, Kolb HJ, Pralle $H$, et al. (1994) Randomized comparison of interferon-alpha with busulfan and hydroxyurea in chronic myelogenous leukemia. The German CML Study Group. Blood 84(12): 4064-4077.

3. Mohammed A Yassin, Ibrahim AA, Hanadi RE, Kamal RA (2007) Beaus lines. Saudi Medical Journal 28(12): 1922-1923.

4. Young HS, Khan ASA, Kendra JR, Coulson IH (2000) The cutaneous side-effects of hydroxyurea. International Journal of laboratory Hematology 22(4): 229-232.

5. Kumar B, Saraswat A, Kaur I (2002) Mucocutaneous adverse effects of hydroxyurea: a prospective study of 30 psoriasis patients. Clin Exp Dermatol 27(1): 813. 